\title{
Humor Styles, Perceived Threat, Funniness of COVID-19 Memes, and Affective Mood in the Early Stages of COVID-19 Lockdown
}

\author{
Jorge Torres-Marín ${ }^{1}(\mathbb{D}) \cdot$ Ginés Navarro-Carrillo ${ }^{2}$ (D) Michael Eid ${ }^{3}$ (D) \\ Hugo Carretero-Dios ${ }^{1}$ iD
}

Accepted: 18 January 2022 / Published online: 5 March 2022

(c) The Author(s), under exclusive licence to Springer Nature B.V. 2022

\begin{abstract}
Existing psychological research has long considered humor as a useful strategy for individuals in coping with adverse circumstances and life stressors. However, empirical studies are called for to better understand the role of humor in facing the COVID-19 pandemic. In a community sample of 527 Spanish adults, we investigated the associations between humor styles, perceived threat from COVID-19, funniness of COVID-19 memes, and individuals' affective mood. Data were collected during the third week of lockdown in Spain (from 26 to 31 March 2020). Our results suggest that intrapsychic humor styles were associated with better coping with the COVID-19 pandemic. Structural equation modelling showed that self-enhancing and aggressive humor styles were related to perceiving COVID-19 as less psychologically threatening, which, in turn, was associated with a greater perceived funniness of COVID-19 memes, thus predicting higher levels of happiness. Interestingly, the opposite pattern of connections was found for self-defeating humor. These findings suggest that humor styles aimed at boosting one's own self, irrespective of their potentially lighter or darker nature, may contribute to alleviating adverse psychological consequences arising from the COVID-19 pandemic.
\end{abstract}

Keywords COVID-19 $\cdot$ Humor styles $\cdot$ Memes $\cdot$ Affective mood $\cdot$ Perceived threat

\section{Introduction}

From late 2019-early 2020, the ongoing pandemic of the novel coronavirus disease (hereafter COVID-19) has dramatically overwhelmed national health systems (e.g., Spain: infections/deaths $=4,977,449 / 86,827$ as of 13 October 2021) and caused major changes in the

Ginés Navarro-Carrillo

gnavarro@ujaen.es

1 Department of Research Methods in Behavioral Sciences, University of Granada, Granada, Spain

Extended author information available on the last page of the article 
daily life of individuals. Faced with COVID-19, individuals have engaged in various behaviors aimed at building resilience to deal with the adverse wide-ranging consequences of this unprecedented situation. Among such resilience practices, the act of making fun of situations triggered by the COVID-19 crisis has extended across a large portion of the population, primarily due to its accessibility (e.g., Internet memes via personal WhatsApp messages). However, exposure to humorous COVID-19 memes still elicits multiple research questions: Are the way people use humor in general and the perceived funniness of COVID19 memes in particular really associated with people's affective mood? Is a positive appreciation of these memes linked to maladaptive forms of humor rather than adaptive ones? Could the way in which people respond to COVID-19 memes be an indirect pathway to account for the relationship between dispositional forms of humor and affective mood during the COVID-19 pandemic? This research was aimed at filling these gaps in the scientific literature.

\subsection{Humor as a Coping Mechanism}

Existing psychological research has long considered humor as a useful strategy for individuals in coping with adverse circumstances and life stressors. In the early 20th century, Sigmund Freud (1905-1994) was a precursor of the tension-release models of humor. He postulated that humor and laughter could be seen as adaptive mechanisms in response to negative emotions such as anger and sadness, as they enable individuals to find some relief contemplating the absurdity and contradiction of the events they experience (i.e., adopting a humorous perspective of one's own life).

Moreover, from the 1980 s, empirical research has progressively reformulated and validated the linkages between humor - as an individual difference variable - and mental health. In a classic study, Martin and Lefcourt (1983) offered preliminary support for the stress-buffering nature of humor, showing that individuals' (increased) use of humor reduced the connection between the presence of stressful life events and mood disturbances. Contemporary research has also consistently reported substantial correlates between humor as a coping mechanism and multiple adaptive psychological outcomes (Kato, 2015; Páez et al., 2013; Puente-Martínez et al., 2018; Schneider et al., 2018, to name but a few). Importantly, it seems that these linkages of humor cannot be generalized to all humor styles but are only found in its adaptive forms (Martin, 2007).

The Humor Styles Model. One of the most prominent frameworks involving the connections between humor (as a trait) and wellbeing is the Humor Styles Model (Martin et al., 2003). This model, unlike other narrower proposals, subsumes four dispositional variables describing how people use adaptive and maladaptive forms of humor in their everyday life. Martin et al. (2003) argued that self-enhancing and aggressive humor styles account for humor-based differences in intrapsychic aspects (e.g., boosting one's own self). Selfenhancing humor represents an adaptive tendency - closely related to prior definitions of humor as a coping strategy - to maintain a benevolent humorous perspective on aversive life circumstances. By contrast, aggressive humor encompasses the inclination to tease and make fun of other individuals without any regrets as a way of denoting a superior status (e.g., putting down others' shortcomings or mistakes). These humorous dispositions are followed by two socially-oriented humor styles (e.g., improving relationships with others) known as affiliative and self-defeating humor styles. Affiliative humor is related to the tra- 
ditionally positive connotation of humor, capturing the use of prosocial and good-natured jokes to promote social cohesion and generate ties with other people (e.g., friends, workmates). Similarly, self-defeating humor is also aimed at obtaining the social approval of others. Yet, this is done by telling offensive jokes about oneself as a way of avoiding (or anticipating) potential criticisms or mockeries from others.

Accumulated empirical evidence has found the Humor Styles Model to be significantly correlated with psychological mood, wellbeing and mental health. Martin et al. (2003) found that, in line with their conceptualization, adaptive humor styles (i.e., affiliative and self-enhancing humor styles) have small-to-moderate negative relationships with traitanxiety and depression and positive relationships with self-esteem-with self-enhancing humor reflecting higher effects sizes. They also revealed that self-defeating humor had the opposite pattern of relationships with wellbeing, and that aggressive humor style seems to be unrelated to these psychological outcomes. Recent meta-analytic extensions of these findings mostly align with these relationships considering various indices of mental health and subjective wellbeing as well as potential sociodemographic and cultural moderating effects (Jiang et al., 2020; Schneider et al., 2018). However, there are mixed results about the benefits of laughing at oneself in certain contexts, which suggests a potential positive side of self-defeating humor (e.g., Heintz \& Ruch, 2018 ${ }^{1}$ ).

Exposure to humorous stimuli. Beyond paradigms that are embedded in differential psychology, an alternative approach to the study of humor and wellbeing has revolved around exposing individuals to various humorous stimuli (e.g., cartoons, verbal jokes, videos). In another classic study, Danzer et al. (1990) scrutinized the effects of humor on negative affective states and observed that hearing a humorous audiotape (vs. non-humorous or control tapes) decreased participants' depression levels after experimentally inducing this negative emotion in them. Multiple studies have provided highly comparable empirical support for this beneficial side of being exposed to (mostly brief) humorous situations in a wide array of wellbeing (Cheng et al., 2019) and mood-related indicators (Slater et al., 2019). Strongly related to our research interest, recent exploratory studies have extended these beneficial effects to exposure to specific online material such as Internet memes, as these stimuli may help to promote a humorous outlook on negative experiences (Akram et al., 2020).

Evidence of direct associations between humor and wellbeing appears to converge across studies and methodological paradigms, thereby validating the assertion of humor as a valid coping mechanism. Nevertheless, further research is needed to ascertain whether these findings are generalizable to a rapidly evolving crisis with deleterious consequences such as the COVID-19 pandemic and to novel humorous material such as COVID-19 memes.

\subsection{COVID-19 and Mental Health}

The unparalleled global collapse triggered by the outbreak of the COVID-19 pandemic, along with generalized preventive nationwide lockdowns, have posed a serious threat to individuals' mental health and continue to do so. This has been empirically proven by a large number of studies. Li et al. (2020) explored ecological behavioral data from an online social network in a group of Chinese individuals. They found differences in levels of posi-

\footnotetext{
${ }^{1}$ Heintz and Ruch (2018) proposed that self-defeating humor may function as a coping strategy for individuals who are more susceptible to negative affective episodes, as they can use self-directed humor to admit their own mistakes or shortcomings and seek social approval, reflecting their humorous side.
} 
tive emotions (e.g., lower happiness and satisfaction with life) and negative emotions (e.g., higher anxiety) between the period before and after the COVID-19 outbreak. Other studies have also found higher levels of post-traumatic stress, anxiety and adjustment disorder symptoms in the Italian general population (Rossi et al., 2020), high rates of loneliness in the United Kingdom (Groarke et al., 2020), anxiety, depression and post-traumatic stress in Spain (González-Sanguino et al. 2020) and acute stress and insomnia symptoms in China (Wang et al., 2021), to name just a few, as a consequence of COVID-19. The negative impact of the COVID-19 pandemic on mental health has also been corroborated by systematic reviews and meta-analytic evidence across diverse geographical areas and populations (Bueno-Notivol et al. 20212 ; Li et al., 2021; Salari et al., 2020).

Despite the growing evidence about the specific impacts of COVID-19 on mental health, the reactions to this adverse scenario are not uniform and cannot be generalized to the entire population. For example, there is evidence of intra-individual variability in people's perceptions of the threat from COVID-19 (Kachanoff et al., 2020) and in their self-reported wellbeing (Zacher \& Rudolph, 2020) during the pandemic. It can therefore be surmised that an adequate use of effective coping mechanisms such as the use of humor to reappraise adverse situations triggered by COVID-19 may be relevant for explaining individual differences in these psychological states.

Humor styles and COVID-19. Some theoretical postulates and empirical evidence support the connection between the use of humor (especially in its adaptive forms) and a better position for coping with COVID-19. According to the conceptualization of adaptive humor styles proposed by Martin et al. (2003), individuals high in self-enhancing humor may be able to re-evaluate threatening events associated with COVID-19 more effectively, maintaining a cheerful but realistic outlook. An increased use of this humor style is correlated with greater optimism (Marrero et al., 2020), greater happiness and life satisfaction (Navarro-Carrillo et al., 2020), lower levels of anxiety and depression (Martin et al., 2003) and a better regulation of negative emotions (Torres-Marín et al., 2018). These empirical correlates are consistent with the notion that self-enhancing humor can contribute to overcoming the obstacles arising from COVID-19. Furthermore, the tendency to create and strengthen social bonds among individuals high in affiliative humor may help them deal with feelings of uncertainty and concern stemming from COVID-19. These individuals also tend to report greater self-perceived social support (Marrero et al., 2020) and lower levels of loneliness (Hampes, 2005). This suggests the presence of valuable interpersonal skills for coping with stressful circumstances arising from COVID-19. Consistent with this, in a very recent study, Olah and Ford (2021) found negative relationships of adaptive humor styles with perceived threat, stress and hopelessness from COVID-19 and positive relationships with protective behaviors such as social distancing during the COVID-19 outbreak.

Regarding the role of maladaptive humor styles, as mentioned, current meta-analytic evidence on aggressive humor suggests that this humor style only makes a negligible contribution to predicting participants' wellbeing (Schneider et al., 2018). Hence, one might expect a non-relevant role of this humor style in coping with the COVID-19 pandemic. Moreover, although self-defeating humor has been proposed as a detrimental humor style for individu-

\footnotetext{
${ }^{2}$ Although these authors found a relatively large increase in depression rates in the general population, they also advised that these findings should be interpreted with caution, since there are considerable variations between the pre- and post-COVID-19 estimated prevalence of depression as a function of the type of methodology used (e.g., self-report vs. clinical diagnosis).
} 
als (Martin et al., 2003), other studies have highlighted its potential positive effect on selfesteem and certain indicators of wellbeing (Heintz \& Ruch, 2018; Navarro-Carrillo et al., 2020). Thus, the use of self-defeating humor as a coping mechanism to deal with COVID-19 should be analyzed in an exploratory manner. Interestingly, the study conducted by Olah and Ford (2021) found that maladaptive humor styles were not significantly related to perceived threat and stress derived from COVID-19 but instead showed meaningful associations with greater hopelessness and lower engagement in protective behaviors.

COVID-19 memes, affective mood, and humor styles. As mentioned earlier, the impact of COVID-19 jokes and memes on wellbeing have received scant attention. Drawing on empirical evidence pertaining to exposure to humor and mental health (e.g., Akram et al., 2020; Slater et al., 2019), it could be inferred that a positive judgment of COVID-19 memes would be associated with a better affective mood. In addition, one could expect that individuals' relatively stable humorous tendencies or humor styles may contribute to shaping specific responses to COVID-19 memes. For instance, a greater engagement in adaptive humor styles may be associated with appreciating humorous COVID-19 stimuli more positively (i.e., as being funnier) on the basis of both an enhanced perspective-taking humor (i.e., reappraisal) and a cheerful/socially playful perspective of life (i.e., share jokes with friends; Martin, 2007; Martin et al., 2003). Recent research has provided evidence that people who tend to face adverse circumstances through humor are more likely to rate COVID humor as funnier and less aversive (Bischetti et al., 2020). Hence, one may argue that individuals high in adaptive humor styles are likely to be more reactive to COVID-19-related humorous signals, showing greater amusement and using such humorous stimuli to maintain a more positive mood (i.e., indirect pathway to a better affective mood).

Regarding maladaptive humor styles, individuals most prone to using aggressive humor - partly due to their lower empathy (Hampes, 2010) — might find certain COVID-19 humorous material funny, particularly stimuli making fun of awkward situations for others. In line with this idea, Bischetti et al. (2020) reported that COVID-19 humor appears to be judged as more aversive compared to neutral humor. We therefore decided to explore the indirect connections between aggressive humor and participants' affective mood via their judgements of COVID-19 memes. Lastly, people high in self-defeating humor may find COVID-19 memes funny as a potential way of laughing at themselves in the face of stressful situations stemming from the pandemic. However, according to the conceptualization of this humor style, this potential positive appreciation of COVID-19 humor may be related to an effort to hide aversive emotional states (Martin, 2007), thus reflecting a negative affective mood.

\subsection{Summary of the Aims and Hypotheses of the Present Research}

We examined the associations between individuals' humor styles and their affective moods in the early stages of the COVID-19 lockdown. We also analyzed whether these associations may be - at least partially_-driven by how people perceived the funniness of humorous COVID-19 memes. Moreover, since humor has proven to be a valid strategy for reevaluating negative events as less menacing (Martin, 2007; Olah \& Ford, 2021), we also considered the perceived COVID-19 threat as an alternative indirect pathway between humor styles and individuals' affective mood in times of COVID-19. We first anticipated that adaptive humor styles would be associated with a lower perceived threat from COVID-19 (H1) and 
better affective mood (H2). Similarly, we hypothesized that a greater funniness of COVID19 memes would correlate with lower perceived COVID-19 threat (H3) and better affective mood (H4). We also expected all humor styles to be positively correlated with the funniness of such memes, with stronger effect sizes for self-enhancing humor (H5). Finally, we tested a theoretical model in which humor styles (especially adaptive ones) were indirectly related to a better affective mood through lower levels of perceived COVID-19 threat and greater perceived funniness of COVID-19 memes (H6).

\section{Method}

\subsection{Participants}

Our sample consisted of 527 adults (347 women [65.8\%], 179 men [34\%], 1 preferred not to state their gender [0.2\%]). Ages ranged from 18 to 68 years $(M=33.99 ; S D=12.87$; Median =30). Of these respondents, $36.1 \%$ were full-time employees, $12.1 \%$ had a parttime job, 34\% were students, $15.4 \%$ were unemployed, and $2.5 \%$ were retired. Respondents were highly educated: $3.4 \%$ had completed a doctorate, $47.1 \%$ held a university degree, $26.2 \%$ had a general certificate of education, $15.4 \%$ had completed vocational training, $4.7 \%$ and $3.0 \%$ reported secondary and primary education, respectively, and 0.2 reported not having any official studies. Sample size was determined based on the recommendations made by Bentler and Chou (1987): there should be at least five individuals per estimated parameter using the maximum likelihood estimator. Because the largest model considered in this investigation had 100 free parameters to be estimated, the required sample size was $5 * 100=500$. Such a sample size also ensured that the power of detecting model misfit was greater than 0.98 for all models considered (with respect to a significance level of $\alpha=0.05$ and the size of model misfit considered by MacCallum et al. [1996], Table 2).

\subsection{Stimuli and Measures}

Battery of COVID-19 memes. This instrument was developed for the present research. As initial stages, four researchers with expertise in humorous material and psychological assessment screened and collected a variety of memes written in Spanish $(N=88)$ from various meme websites (e.g., www.memedroid.com), social media (e.g., Twitter), Internet fora and cross-platform instant messaging applications (e.g., WhatsApp). To fine-tune this list down to a manageable number of memes and guarantee sufficient intra-stimuli variability, the eligibility criteria for memes were as follows: (1) capturing humorous exaggerations of situations deriving from the COVID-19 pandemic (e.g., panic buying, adaptation to teleworking, walking the dog excessively or gaining weight during the lockdown); (2) involving a benign humorous view of the COVID-19 pandemic, avoiding sensitive issues for society (e.g., deaths, health-care disasters, financial problems, people socially/physically isolated or seriously ill); and (3) avoiding direct or indirect mentions to political and religious messages. After discussing and agreeing on potential divergences on the selection, this process led to a total of 10 eligible memes for their further utilization in this research (see OSF link for Supplemental Material). The funniness of each meme was rated from 1 (not at all funny) to 5 (very funny). 
Humor Styles Questionnaire. This measure consists of 32 items that assess the four humor styles (Martin et al., 2003; Spanish version by Torres-Marín et al., 2018): (1) affiliative humor (8 items; e.g., "I enjoy making people laugh"); (2) self-enhancing humor (8 items; e.g., "If I am feeling depressed, I can usually cheer myself up with humor"); (3) aggressive humor ( 8 items; e.g., "If someone makes a mistake, I will often tease them about it"); and (4) self-defeating humor ( 8 items; e.g., "I let people laugh at me or make fun at my expense more than I should"). Responses are given on a 7-point rating scale ( $1=$ strongly disagree; $7=$ strongly agree). The Spanish version of the HSQ shows a stable four-factor structure and relatively large coefficients of internal consistency, good nomological validity, and adequate applicability to different environments/populations (Navarro-Carrillo et al., 2020; Torres-Marín et al., 2018).

Perceived threat from COVID-19. We assessed respondents' perceived psychological threat (i.e., feelings of risk, worry, uncertainty) in the context of the COVID-19 pandemic by adapting the Financial Threat Scale (Marjanovic et al., 2013) to the scenario of the unfolding COVID-19 pandemic crisis in Spain (i.e., "Taking into account the situation triggered by COVID-19, please report how you feel by answering the following questions"). Analogous approaches have been used in recent studies (e.g., Navarro-Carrillo et al., 2021). This measure consists of a total of 5 items (e.g., "How much do you feel at risk?"). Respondents answered on a 5-point rating scale ranging from 1 (not at all) to 5 (a great deal).

Scale for mood assessment. This measure comprises 16 items that assess transient positive and negative affective states through four subscales (Sanz, 2001): happiness (4 items; e.g., "I feel joyful"), sadness-depression (4 items; e.g., "I feel sad"), anxiety (4 items; e.g., "I feel anxious"), and anger-hostility (4 items; e.g., "I feel angry"). This measure has shown good internal consistency, adequate factorial validity and high correspondence with other mood-related scales (Sanz, 2001). Respondents answered on an 11-point rating scale ranging from 1 (not at all) to 11 (very much). Since the scores of all the negative affect subscales were highly intercorrelated, we computed a global dimension named distress by aggregating their scores.

\subsection{Procedure}

The sample was obtained through online recruitment using the Qualtrics survey software platform. Data were collected for six days, from 26 to 31 March 2020 (i.e., the third week of lockdown in Spain). The study was advertised as an online survey on the role of humor in the ongoing COVID-19 pandemic through social media (i.e., Instagram, Facebook and Twitter) and cross-platform instant messaging applications (i.e., WhatsApp and Telegram). Instructions included a brief description of the study and the estimated time to complete the survey ( $\approx 10 / 15 \mathrm{~min}$ ). The inclusion criterion was being at least 18 years of age and having Spanish citizenship. Respondents gave written informed consent before starting the online survey. We guaranteed the anonymity and confidentiality of participants' responses and emphasized that participation was voluntary. This research was conducted in accordance with the ethical guidelines of the local university and with the Ethical Standards of the 1964 Declaration of Helsinki. 


\subsection{Statistical Analyses}

Descriptive statistics (i.e., mean and standard deviations) and internal consistencies coefficients were calculated for all the research variables. We also calculated zero-order and partial correlations (i.e., controlling for age and gender) between humor styles, perceived threat from COVID-19 (or COVID-19 Threat), perceived funniness of COVID-19 memes (or COVID-19 Fun), and individuals' affective mood (comprising Distress and Happiness).

In a subsequent step, structural equation modeling (SEM) was applied with Mplus 8.1 software (Muthén \& Muthén, 1998-2017) to analyze the indirect effects (IEs) of humor styles on Distress and Happiness through COVID-19 Threat and COVID-19 Fun. The robust maximum likelihood (MLR) estimator was used to estimate the model. Three indicators were used for each latent variable. For COVID-19 Fun and the four humor styles, which were all unidimensional constructs, three parcels were created as indicators for each of them (Little et al., 2013). Regarding Distress, the average scores of the anxiety, sadness, and hostility factors were used as indicators. Finally, COVID-19 Threat (assessed by five items) and Happiness (assessed by four items) included the three most representative items as indicators (i.e., higher discrimination indices ${ }^{3}$ ).

We assessed model fit using the root mean square error of approximation (RMSEA), the standardized root-mean square residual (SRMR), the comparative fit index (CFI), and the Tucker-Lewis index (TLI). RMSEA and SRMR values less than 0.05 and CFI and TLI values greater than or equal to 0.95 were considered evidence of good fit (Schumacker \& Lomax 2010). First, the measurement model was tested with the MLR estimator for the eight latent constructs of our study (i.e., Affiliative humor, Aggressive humor, Selfenhancing humor, Self-defeating humor, COVID-19 Threat, COVID-19 Fun, Happiness and Distress). Next, three structural models were tested: (A) Model 1 tested the direct effects

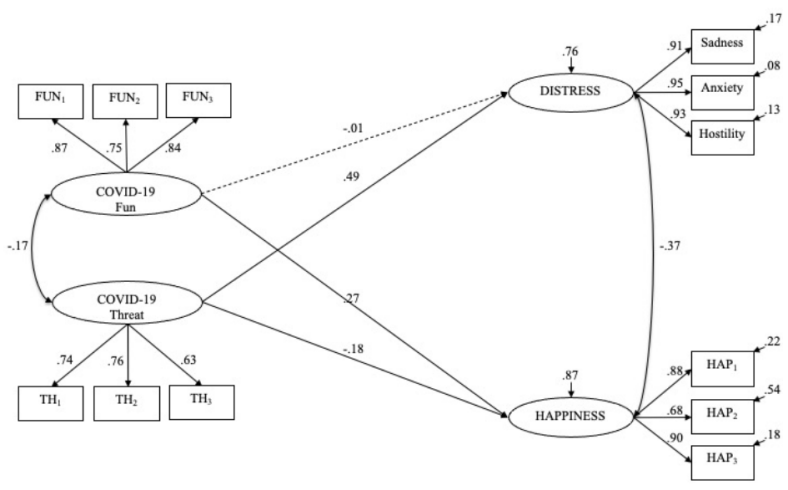

Fig. 1 The Structural Equation Model Testing the Direct Effects of COVID-19 Threat and COVID-19 Fun on Distress and Happiness (Model 1). Note: Solid lines indicate significant paths, and dotted lines indicate nonsignificant paths ( $p=.05$, two-sided tests). Standardized coefficients are reported. The arrows above the latent variables represent the residual variance for the dependent variables. All non-standardized parameters with standard errors and standardized parameters are in the OSF link for Supplemental Material

\footnotetext{
${ }^{3}$ As the number of items discouraged the application of the parceling technique, we selected the three items of each subscale with the higher item-total correlations. This approach enabled us to include the individual items that appeared to optimize the measurement of the same unitary construct.
} 


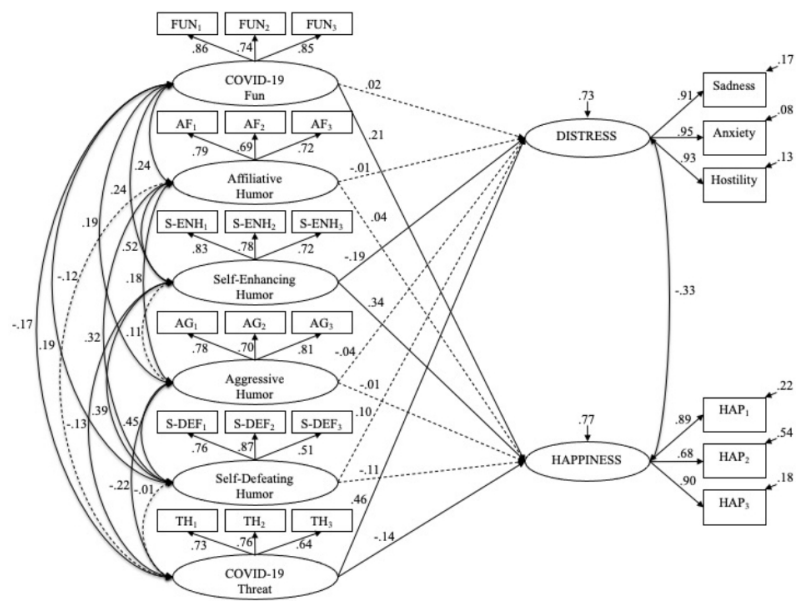

Fig. 2 The Structural Equation Model Testing the Direct Effects of Humor Styles, COVID-19 Threat, and COVID-19 Fun on Distress and Happiness (Model 2). Note: Solid lines indicate significant paths, and dotted lines indicate nonsignificant paths ( $p=.05$, two-sided tests). Standardized coefficients are reported. The arrows above the latent variables represent the residual variance for the dependent variables. All non-standardized parameters with standard errors and standardized parameters are in the OSF link for Supplemental Material

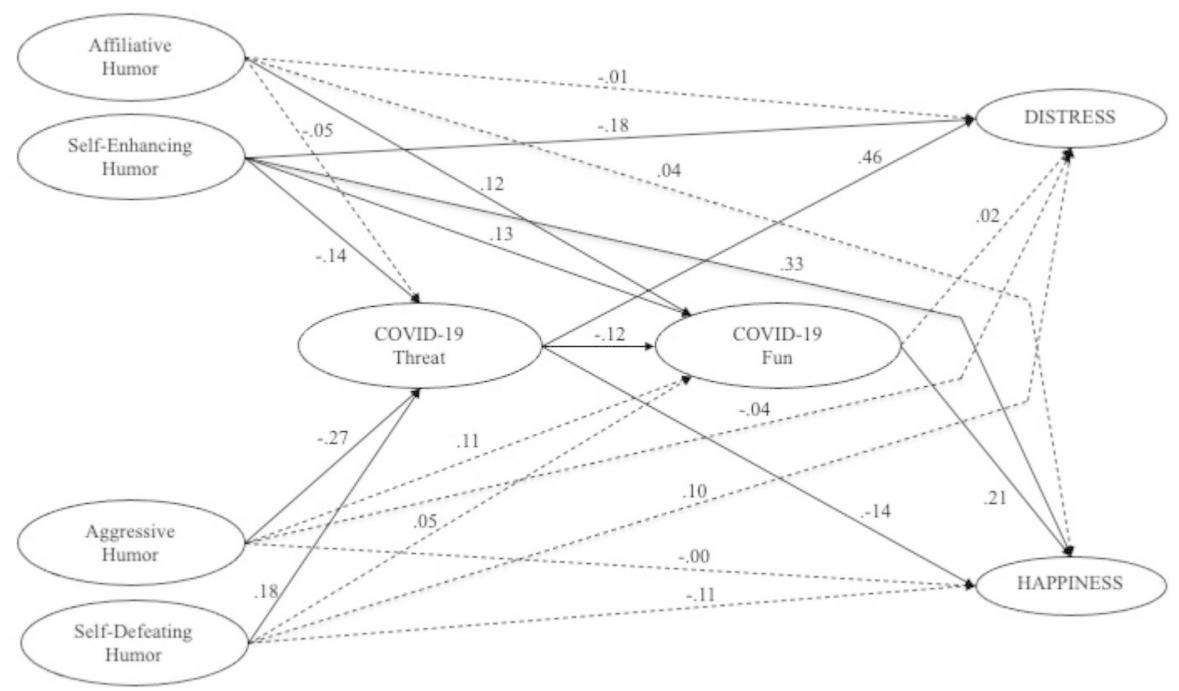

Fig. 3 The Structural Equation Model Testing the Indirect Effects of Humor Styles on Distress and Happiness through COVID-19 Threat and COVID-19 Fun (Model 3). Note: Solid lines indicate significant paths, and dotted lines indicate nonsignificant paths ( $p=.05$, two-sided tests). Standardized coefficients are reported. Indicators of each latent variable, the correlations between independent variables and between the residuals of distress and happiness are not shown in the figure for parsimony 
of COVID-19 Threat and COVID-19 Fun on Distress and Happiness (Fig. 1); (B) Model 2 tested the preceding model including the humor styles to examine their direct effects on Distress and Happiness (Fig. 2); finally, (C) Model 3 tested the direct associations of humor styles with Distress and Happiness and their potential indirect pathways via COVID-19 Threat and COVID-19 Fun (Fig. 3). Before testing Model 3, we analyzed the interaction effects between humor styles and COVID-19 Fun, the interaction between humor styles and COVID-19 Threat, and the interaction between COVID-19 Fun and COVID-19 Threat. We used bootstrapping to examine the significance of IEs (Preacher \& Hayes 2008). If the 95\% (bias-corrected and accelerated) confidence intervals (CIs) did not include 0 , then the IEs were significant. 1000 bootstrap samples were used to estimate the CIs.

\section{Results}

\subsection{Descriptive and Correlation Analyses}

Preliminary analyses. Descriptive statistics and internal consistency coefficients for all measurements are presented in Table 1. Mean scores and standard deviations reflected sufficient response variability and all measures showed appropriate internal consistency (Cronbach's $\alpha \mathrm{s} \geq 0.75$ ). Gender- and age-based differences can be seen in the OSF link for Supplemental Material (Table S1). Aggressive and Self-defeating humor styles were more pronounced in men, while women reported greater Distress. Older age was slightly correlated with Self-enhancing humor.

Correlation analyses. Table 1 also shows the bivariate and partial correlations (controlling for age and gender) between humor styles, COVID-19 Threat, COVID-19 Fun, and affective mood. Among humor styles, Affiliative humor was unrelated to COVID-19 Threat but was related to greater Happiness; Self-enhancing humor was related to lower levels of COVID-19 Threat and Distress, and also to higher Happiness; Aggressive humor was related to lower COVID-19 Threat and unrelated to affective mood; and Self-defeating humor was unrelated to both COVID-19 Threat or the mood dimensions. As expected, all these humor styles were positively correlated with COVID-19 Fun. COVID-19 Threat was associated with reduced COVID-19 Fun, and, interestingly, this latter variable was positively linked with Happiness. Finally, COVID-19 Threat was also related to diminished Happiness and increased Distress.

\subsection{Measurement Model}

To determine whether the measurement model fit the data adequately, a confirmatory factor analysis (CFA) was conducted. The MLR estimation method was used to estimate the model. Eight latent variables were included in the measurement model (i.e., Affiliative humor, Aggressive humor, Self-enhancing humor, Self-defeating humor, COVID-19 Threat, COVID-19 Fun, Happiness, and Distress). Results showed that the measurement model fit the data well: $\chi^{2}(224, N=527)=380.27, p<.001$; RMSEA $=0.036$; $\operatorname{SRMR}=0.038$; $\mathrm{CFI}=0.971$; and $\mathrm{TLI}=0.962$. The standardized factor loadings of all the indicators on the latent variables were significant (loadings $\geq 0.51, p s<.001$ ). These results showed that the measurement model was an appropriate starting point for further analyses. 


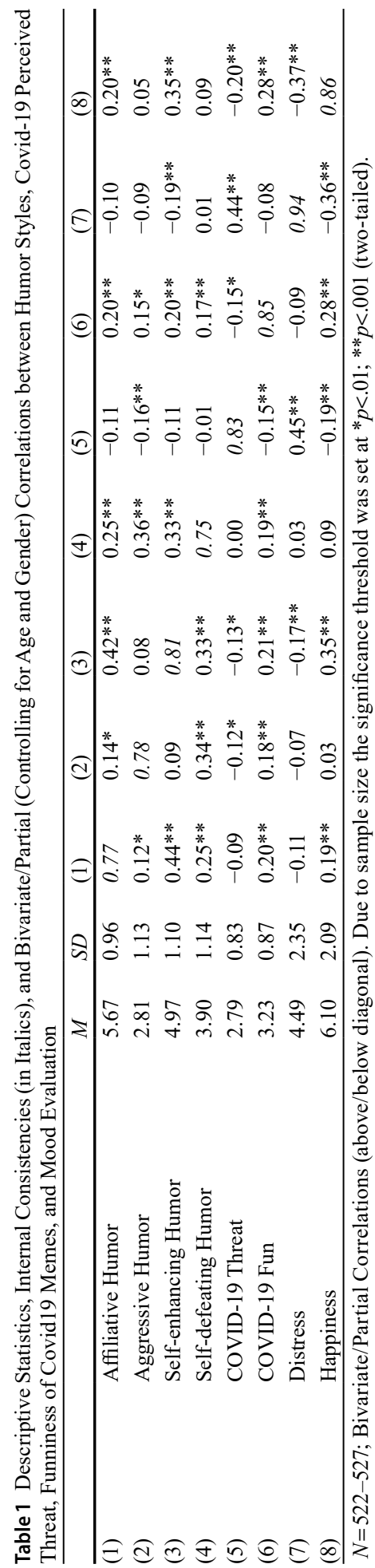




\subsection{Structural Equation Modelling}

In the first place, two models were tested in which no IEs were modeled (Models 1 and 2).

COVID-19 Threat and COVID-19 Fun. Model 1 tested the direct associations of COVID-19 Threat and COVID-19 Fun with Distress and Happiness. This model showed a good fit to the data, namely: $\chi^{2}(48, N=527)=84.74, p<.001, \mathrm{CFI}=0.988, \mathrm{TLI}=0.983$, RMSEA $=0.038, \mathrm{SRMR}=0.038$. As can be seen in Fig. 1 , the path coefficient from COVID19 Threat to Distress was positive and significant $(\beta=0.49, p<.001)$. Conversely, COVID19 Fun did not show a significant path with this negative mood indicator. Furthermore, the path coefficients from COVID-19 Threat $(\beta=-0.18, p=.001)$ and COVID-19 Fun $(\beta=0.27$, $p<.001)$ to Happiness were both significant.

The inclusion of humor styles. Model 2 included humor styles to examine their direct effects on Distress and Happiness. This model also showed an adequate fit to the data: $\chi 2(224, N=527)=380.271, p<.001, \mathrm{CFI}=0.971, \mathrm{TLI}=0.965, \mathrm{RMSEA}=0.036$, $\mathrm{SRMR}=0.038$. As Fig. 2 illustrates, all humor styles yielded significant positive paths to COVID-19 Fun (coefficients ranging between 0.19 and 0.24) and COVID-19 Fun was associated with greater Happiness $(\beta=0.21, p<.001)$. Whereas non-significant paths from Affiliative and Self-defeating humor styles to COVID-19 Threat emerged, both Aggressive humor $(\beta=-0.22)$ and Self-enhancing humor $(\beta=-0.13)$ styles were negatively associated with COVID-19 Threat. This latter variable showed a weak negative path to Happiness $(\beta=-0.14, p=.01)$ and a strong positive path to Distress $(\beta=0.46, p<.001)$. As regards the relationships between humor styles and mood indicators, only Self-enhancing humor was a significant predictor, showing a negative path to Distress $(\beta=-0.19, p=.01)$ and a positive path to Happiness $(\beta=0.34, p<.001)$.

Moderation effects. After identifying the direct effects of humor styles, COVID-19 Fun and COVID-19 Threat on Distress and Happiness, we analyzed the moderation effects between these predictors. We introduced each of the potential interactions one by one into Model 2. As can be seen in the OSF link for Supplemental Material (Table S2), no interaction effects emerged.

Indirect effects of humor styles on affective mood. Model 3 analyzed the direct associations of humor styles with Distress and Happiness, while also accounting for COVID-19 Threat and COVID-19 Fun (Fig. 3; Tables 2 and 3). This model showed an acceptable fit to the data: $\chi 2(224, N=527)=415.599, p<.001, \mathrm{CFI}=0.969$, TLI=0.962, RMSEA=0.040, $\mathrm{SRMR}=0.038$.

Affiliative humor. This adaptive humor style did not have a total IE on Distress through COVID-19 Threat and COVID-19 Fun (total IE $=-0.02,95 \%$ CI $[-0.09,0.04]$ ). Bootstrapping indicated that Affiliative humor did not have significant specific IEs on Distress through COVID-19 Threat (specific IE $=-0.02,95 \%$ CI $[-0.09,0.03]$ ), COVID-19 Fun (specific $\mathrm{IE}=0.00,95 \% \mathrm{CI}[-0.01,0.02])$ or considering both COVID-19 Threat and COVID-19 Fun as serial predictors (specific IE $=0.00,95 \% \mathrm{CI}[-0.00,0.00]$ ). Furthermore, although Affiliative humor showed a total IE on Happiness through COVID-19 Threat and COVID19 Fun (total IE $=0.03,95 \%$ CI $[0.00,0.07]$ ), bootstrapping only showed a significant role of COVID-19 Fun (specific IE $=0.03,95 \%$ CI $[0.00,0.05]$ ) in explaining the connection between Affiliative humor and Happiness. Thus, Affiliative humor had a positive connection with COVID-19 Fun $(\beta=0.12, p=.04)$, which in turn was positively associated with Happiness $(\beta=0.21, p<.001)$. Interestingly, neither COVID-19 Threat (specific IE $=0.01$, 
Table 2 Indirect Effects on Distress and 95 Percent Confidence Intervals for Model 3 (Fig. 3).

\begin{tabular}{|c|c|c|c|}
\hline & \multirow[b]{2}{*}{$\begin{array}{l}\text { Esti- } \\
\text { mated } \\
\text { effect }\end{array}$} & \multicolumn{2}{|c|}{$95 \% \mathrm{CI}$} \\
\hline & & $\begin{array}{l}\text { Lower } \\
\text { bonds }\end{array}$ & $\begin{array}{l}\text { Upper } \\
\text { bonds }\end{array}$ \\
\hline Affiliative Humor Total Indirect Effect & -.02 & -.09 & .04 \\
\hline Affiliative $\rightarrow$ COVID-19 Threat $\rightarrow$ Distress & -.02 & -.09 & .03 \\
\hline Affiliative $\rightarrow$ COVID-19 Fun $\rightarrow$ Distress & .00 & -.01 & .02 \\
\hline Affiliative $\rightarrow$ COVID-19 Threat $\rightarrow$ COVID-19 Fun $\rightarrow$ Distress & .00 & .00 & .00 \\
\hline Self-Enhancing Humor Total Indirect Effect & -.06 & -.13 & .00 \\
\hline Self-Enhancing $\rightarrow$ COVID-19 Threat $\rightarrow$ Distress & -.06 & -.13 & -.00 \\
\hline Self-Enhancing $\rightarrow$ COVID-19 Fun $\rightarrow$ Distress & .00 & -.01 & .02 \\
\hline Self-Enhancing $\rightarrow$ COVID-19 Threat $\rightarrow$ COVID-19 Fun $\rightarrow$ Distress & .00 & -.00 & .00 \\
\hline Aggressive Humor Total Indirect Effect & -.12 & -.18 & -.07 \\
\hline Aggressive $\rightarrow$ COVID-19 Threat $\rightarrow$ Distress & -.13 & -.18 & -.07 \\
\hline Aggressive $\rightarrow$ COVID-19 Fun $\rightarrow$ Distress & .00 & -.01 & .02 \\
\hline Aggressive $\rightarrow$ COVID-19 Threat $\rightarrow$ COVID-19 Fun $\rightarrow$ Distress & .00 & -.00 & .00 \\
\hline Self-Defeating Humor Total Indirect Effect & .08 & .03 & .14 \\
\hline Self- Defeating $\rightarrow$ COVID-19 Threat $\rightarrow$ Distress & .08 & .03 & .14 \\
\hline Self-Defeating $\rightarrow$ COVID-19 Fun $\rightarrow$ Distress & .00 & -.00 & .01 \\
\hline $\begin{array}{l}\text { Self-Defeating } \rightarrow \text { COVID-19 Threat } \rightarrow \text { COVID-19 Fun } \rightarrow \\
\text { Distress }\end{array}$ & .00 & -.00 & .00 \\
\hline COVID-19 Threat $\rightarrow$ COVID-19 Fun $\rightarrow$ Distress & -.00 & -.00 & .01 \\
\hline
\end{tabular}

Note: $95 \%$ CI $=$ The $95 \%$ Confidence Interval.

95\% CI $[-0.01,0.05])$ nor this variable along with COVID-19 Fun as joint predictors (specific $\mathrm{IE}=0.00,95 \% \mathrm{CI}[-0.01,0.01])$ played a significant role in explaining the association between Affiliative humor and Happiness.

Self-enhancing humor. No significant total IE of Self-enhancing humor on Distress through COVID-19 Threat and COVID-19 Fun was found (total IE $=-0.06,95 \%$ CI $[-0.13$, 0.09]). However, bootstrapping indicated a significant role of COVID-Threat in explaining the association between Self-enhancing humor and Distress (specific IE $=-0.06,95 \% \mathrm{CI}$ $[-0.13,-0.00])$. Self-enhancing humor was negatively associated with COVID-19 Threat $(\beta=-0.14, p=.03)$, which was related to lower levels of Distress $(\beta=0.46, p<.001)$. The remaining potential specific IEs between Self-enhancing humor and Distress were non-significant (Table 2). Regarding Happiness, a total IE of Self-enhancing humor on Happiness emerged (total IE $=0.05,95 \% \mathrm{CI}[0.02,0.09]$ ). This humor style had a significant IE on Happiness through COVID-19 Threat (specific IE $=0.02,95 \%$ CI $[0.00,0.05]$ ). Self-enhancing humor was negatively associated with COVID-19 Threat, which in turn was related to increased Happiness $(\beta=-0.14, p=.01)$. A significant IE also emerged through COVID-19 Fun (specific IE $=0.03,95 \%$ CI $[0.01,0.06])$. Self-enhancing humor was positively associated with COVID-19 Fun $(\beta=0.13, p=.04)$, which, in this case, was related to higher levels of Happiness $(\beta=0.21, p<.001)$. More importantly, this intrapsychic humor style also yielded an IE on Happiness through COVID-19 Threat and COVID-19 Fun (specific $\mathrm{IE}=0.01,95 \% \mathrm{CI}[0.00,0.01])$. In short, Self-enhancing humor had a negative association with COVID-19 Threat $(\beta=-0.14, p=.03)$, which in turn was linked to increased COVID19 Fun $(\beta=-0.12, p=.04)$, and finally to greater Happiness levels $(\beta=0.21, p<.001)$. 
Table 3 Indirect Effects on Happiness and 95 Percent Confidence Intervals for Model 3 (Fig. 3 ).

Affiliative Humor Total Indirect Effect

Affiliative $\rightarrow$ COVID-19 Threat $\rightarrow$ Happiness

Affiliative $\rightarrow$ COVID-19 Fun $\rightarrow$ Happiness

Affiliative $\rightarrow$ COVID-19 Threat $\rightarrow$ COVID-19 Fun $\rightarrow$

Happiness

Self-Enhancing Humor Total Indirect

Effect

Self-Enhancing $\rightarrow$ COVID-19 Threat $\rightarrow$ Happines

Self-Enhancing $\rightarrow$ COVID-19 Fun $\rightarrow$ Happiness

Self-Enhancing $\rightarrow$ COVID-19 Threat $\rightarrow$ COVID-19 Fun $\rightarrow$ Happiness

Aggressive $\rightarrow$ COVID-19 Fun $\rightarrow$ Happiness

Aggressive $\rightarrow$ COVID-19 Threat $\rightarrow$ COVID-19 Fun $\rightarrow$

Happiness

Self-Defeating Humor Total Indirect

Effect

Self- Defeating $\rightarrow$ COVID-19 Threat $\rightarrow$ Happiness

Self-Defeating $\rightarrow$ COVID-19 Fun $\rightarrow$ Happiness

Self-Defeating $\rightarrow$ COVID-19 Threat $\rightarrow$ COVID-19 Fun $\rightarrow$ Happiness

COVID-19 Threat $\rightarrow$ COVID-19 Fun $\rightarrow$ Happiness
Aggressive Humor Total Indirect Effect

Aggressive $\rightarrow$ COVID-19 Threat $\rightarrow$ Happiness

\begin{tabular}{|c|c|c|}
\hline \multirow[b]{2}{*}{$\begin{array}{l}\text { Estimated } \\
\text { effect }\end{array}$} & \multicolumn{2}{|l|}{$95 \% \mathrm{CI}$} \\
\hline & $\begin{array}{l}\text { Lower } \\
\text { bonds }\end{array}$ & Upper bonds \\
\hline .03 & .00 & .07 \\
\hline .01 & -.01 & .05 \\
\hline .03 & .00 & .05 \\
\hline .00 & -.01 & .01 \\
\hline .05 & .02 & .09 \\
\hline .02 & .00 & .05 \\
\hline .03 & .01 & .06 \\
\hline .01 & .00 & .01 \\
\hline .07 & .04 & .12 \\
\hline .04 & .02 & .08 \\
\hline .02 & .00 & .06 \\
\hline .01 & .00 & .02 \\
\hline-.02 & -.06 & .01 \\
\hline-.03 & -.05 & -.01 \\
\hline .01 & -.01 & .04 \\
\hline-.00 & -.01 & -.00 \\
\hline-.03 & -.05 & -.01 \\
\hline
\end{tabular}

Note: $95 \% \mathrm{CI}=$ The $95 \%$ Confidence Interval.

Aggressive humor. This humor style showed a total IE on Distress (total IE $=-0.12$, $95 \% \mathrm{CI}=(-0.18,-0.07)$. We found a significant role of COVID-19 Threat in explaining the relationship between Aggressive humor and Distress (IE $=-0.13,95 \%$ CI $[-0.18$, $-0.07])$. Aggressive humor was associated with reduced COVID-19 Threat $(\beta=-0.27, p<$ $.001)$, which in turn was related to decreased Distress $(\beta=0.46, p<.001)$. By contrast, there were no significant IEs of Aggressive humor on Distress through COVID-19 Fun (specific $\mathrm{IE}=0.00,95 \% \mathrm{CI}[-0.01,0.02])$, or COVID-19 Threat and COVID-19 Fun as serial predictors (specific IE $=0.00,95 \% \mathrm{CI}[-0.00,0.00]$ ), as COVID-19 Fun was unrelated to Distress $(\beta=0.02, p=.76)$. Aggressive humor also had a total IE on Happiness (total IE $=0.07,95 \%$ CI $[0.04,0.12])$. COVID-19 Threat played a significant role in explaining the association between this maladaptive style of humor and Happiness (specific IE $=0.04,95 \%$ CI $[0.02$, 0.08]). Aggressive humor was negatively associated with COVID-19 Threat, which in turn was related to increased Happiness $(\beta=-0.14, p=.01)$. A significant IE was also found through COVID-19 Fun (specific IE $=0.02,95 \%$ CI $[0.00,0.06]$ ). Aggressive humor was positively associated with COVID-19 Fun $(\beta=0.11, p=.08)$, which in turn was associated with greater levels of Happiness $(\beta=0.21, p<.001)$. As found for Self-enhancing humor, Aggressive humor yielded an IE on Happiness through COVID-19 Threat and COVID-19 Fun (specific IE $=0.01,95 \% \mathrm{CI}[0.00,0.02]$ ). Aggressive humor was negatively related to COVID-19 Threat $(\beta=-0.27, p<.001)$, which was associated with enhanced COVID-19 Fun $(\beta=-0.12, p=.04)$, and finally with greater Happiness $(\beta=0.21, p<.001)$. 
Self-defeating humor. COVID-19 Threat and COVID-19 Fun had a significant role in explaining the link between Self-defeating humor and Distress (total IE $=0.08,95 \%$ CI $[0.03,0.14])$. We found a significant IE of COVID-Threat in the association between Self-defeating humor and Distress (specific IE $=0.08,95 \%$ CI $[0.03,0.14]$ ). Self-defeating humor was positively associated with COVID-19 Threat $(\beta=0.18, p=.02)$, which in turn was related to amplified Distress $(\beta=0.46, p<.001)$. There were no significant IEs of Selfdefeating humor on Distress through COVID-19 Fun (specific IE $=0.00,95 \%$ CI $[-0.00$, 0.01]), or COVID-19 Threat and COVID-19 Fun as serial predictors (specific IE $=0.00$, $95 \%$ CI $[-0.00,0.01])$. The reason for this was that, as mentioned, COVID-19 Fun was unrelated to Distress $(\beta=0.02, p=.76)$. Considering Happiness as an outcome variable, no significant total IEs of Self-defeating humor on Happiness emerged (total IE $=-0.02,95 \%$ CI $[-0.06,0.01])$. Although COVID-19 Fun did not account for the relationship between Self-defeating humor and Happiness (specific IE $=0.01,95 \%$ CI $[-0.01,0.04]$ ) - this humor style was unrelated to COVID-19 Fun $(\beta=0.05, p=.51)$ - COVID-19 Threat showed a significant role in accounting for the connection between Self-defeating humor and Happiness (specific IE $=-0.03,95 \%$ CI $[-0.05,-0.01]$ ). Self-defeating humor was positively associated with COVID-19 Threat $(\beta=0.18, p=.02)$, which in turn was linked to lower levels of Happiness $(\beta=-0.14, p=.01)$. Interestingly, an indirect pathway through COVIDThreat and COVID-Fun emerged (specific IE $=-0.00,95 \%$ CI $[-0.01,-0.00]$ ). Self-defeating humor was related to higher COVID-19 Threat $(\beta=0.18, p=.02)$, which was associated with lower COVID-19 Fun ( $\beta=-0.12, p=.04)$; this, in turn, was related to reduced Happiness $(\beta=0.21, p<.001)$.

\section{Discussion}

This research contributes to filling a gap in the literature concerning the role of humor in the early stages of the COVID-19 pandemic. Four major findings emerged: (1) although, as expected, adaptive humor styles correlated with a better affective mood, (2) only intrapsychic forms of humor (i.e., self-enhancing and aggressive humor) were significantly and negatively related to COVID-19 perceived threat. Moreover, (3) an elevated funniness of COVID-19 memes yielded positive associations with a better affective mood, less perceived COVID-19 threat, and a high use of all humor styles. Finally, (4) the SEM analysis revealed that perceived COVID-19 threat and the perceived funniness of COVID-19 memes, either individually or serially, accounted for the associations between humor styles and affective mood. In short, intrapsychic humor styles were associated with reduced perceived threat from COVID-19, which, in turn was related to a greater perceived funniness of COVID-19, thus predicting increased happiness. Interestingly, the opposite pattern of connections was found for self-defeating humor.

Our expectations about the positive impact of adaptive humor styles on participants' mood by linking these tendencies to a decreased COVID-19 threat (H1) were only partially met. Correlation analyses showed that only the use of self-enhancing humor had a negative correlation - although of small effect size - with this construct. One could reasonably argue that high scorers in self-enhancing humor would be more likely to find humorous outlooks in aversive situations triggered by COVID-19 (e.g., observing and imaging good-natured absurdities and contradictions derived from the pandemic), which, in turn, could lead them 
to be less pessimistic and perceive the COVID-19 pandemic as less threatening (Martin, 2007; Olah \& Ford, 2021). An alternative explanation would be that an increased use of self-enhancing humor could be part of a set of personal skills/strengths that may be effective to cope with COVID-19. This aligns well with both the empirical relationship between this humor style and optimism (Marrero et al., 2020) as well as the overlapping variance between humor (as a coping mechanism) and multiple character strengths, such as bravery or hope (Müller \& Ruch, 2011). By contrast, affiliative humor appeared to be unrelated to COVID-19 perceived threat. This suggests that the prosocial tendency to share funny things/jokes with others (e.g., send humorous internet memes) is not relevant enough for predicting individual differences in the perceived threat from COVID-19.

Unexpectedly, aggressive humor was associated with a less threatening view of the pandemic. Based on the conceptualization of Martin et al. (2003), one might argue that individuals high in aggressive humor will tend to make fun of others' adverse - even dramatic - situations (e.g., those unleashed by the COVID-19) as a way of enhancing their own self. Although empirical findings suggest that this hostile humorous strategy is not effective for improving their wellbeing (Schneider et al., 2018), our results suggest that a regular use of hostile forms of humor might contribute to perceiving COVID-19 as less psychologically threatening. Finally, the correlational analysis did not show any relationship between selfdefeating humor and perceived COVID-19 threat.

Regarding our hypothesis about the direct associations between humor styles and indicators of affective mood in times of COVID-19 (H2), our findings widely replicated and extended earlier empirical data. Adaptive humor styles, especially self-enhancing humor, were most conducive to having greater scores in increased happiness and lower scores in personal distress, reflecting small-to-moderate effect sizes. Interestingly, however, greater effects sizes emerged for happiness (positive affect) than for distress (negative affect), which converges well with other personality-affectivity relationships (e.g., Mendiburo-Seguel et al., 2015). Moreover, no significant direct associations were found between aggressive and self-defeating humor styles and any of the measures of individuals' mood. Taken together, these results are aligned with prior studies according to which self-enhancing humor is the main predictor of individual differences in wellbeing (Navarro-Carrillo et al., 2020; Schneider et al., 2018).

When examining the associations of funniness of COVID-19 memes with COVID-19 threat (H3), affective mood (H4), and humor styles (H5), we observed that individuals who judged COVID-19 memes as funnier tended to perceive the COVID-19 pandemic as less threatening and to report higher levels of happiness and a greater use of all humor styles (reflecting highly comparable effect sizes). These findings suggest that a more positive appreciation of COVID-19 memes is more frequent among individuals who cognitively estimate a lower likelihood of being affected by the COVID-19 pandemic. A potential explanation of this finding could be drawn from the Construal-Level Theory of the Psychological Distance (see Trope and Liberman, 2010). Presumably, individuals who are more likely to perceive the COVID-19 pandemic as not directly affecting themselves but others (i.e., self vs. others) are in a better position to enjoy this type of humorous stimuli (in this case, Internet memes) inspired by the tragic circumstances arising from COVID-19. Previous findings about the appreciation of COVID-19-related humor have already shown that spatial/psychological distance can modulate the perceived funniness of such humorous stimuli; people who lived at a greater distance from the first epicenter of the pandemic perceived greater 
funniness (Bischetti et al., 2020). Although this latter research did not find a relevant role for social psychological distance (i.e., perceived risk) in the funniness of COVID-19 humor, our findings suggest that this cognitive separation (i.e., self vs. others) can be relevant for explaining intra-individual variability in the perceived funniness of COVID-19 humor.

A greater perceived funniness of COVID-19 memes was also associated with a more positive affective mood. This association is consistent with the beneficial impacts of exposure to humor-related stimuli on wellbeing (e.g., Cheng et al., 2019). In general, this finding supports the notion that COVID-19 memes, by means of reproducing social experiences, symbolic values and feelings related to this crisis, represent a humor-based collective response that can mitigate emotional stressful circumstances related to the pandemic (Flecha-Ortiz et al., 2021; Páez \& Pérez, 2020). Even though COVID-19-related humor has been generally categorized and judged as more aversive than non-COVID-19 humor (Bischetti et al., 2020), our study suggests that the ability to enjoy these stimuli seems to be related to higher self-reported happiness. Finally, the greater perceived funniness of COVID-19 humor had positive correlations with all humor styles. One might argue that the participants' tendencies to generate and appreciate positively humor (vs. humorlessness) are conductive to a greater enjoyment of COVID-19 memes, irrespective of the underlying motivation of the different dispositional humor variables (i.e., adaptive vs. maladaptive).

Crucially for our main goals, SEM analyses also showed that the perceived funniness of COVID-19 memes and the perceived threat from COVID-19 can be considered as indirect pathways of some of the connections between humor styles and affective mood. The results of self-enhancing humor were as expected (H6). Individuals who are more likely to have a humorous outlook of adverse situations tend to perceive COVID-19 as less threatening, which subsequently predicts a higher perceived funniness of COVID-19 memes and thus a better affective mood. Lower levels of perceived threat from COVID-19 also contributed to predicting lower personal distress; however, no relevant role of the funniness of COVID-19 memes was found when predicting negative affect. Presumably, the tendency to maintain a serene and funny perspective on their life leads individuals high in self-enhancing humor to judge COVID-19 as less-threatening and, in turn, this is likely to promote a more positive evaluation of COVID-19 memes. Such individuals may be more reactive to the humorous side of the absurdities and contradictions of this pandemic, which is likely to help them to have a more positive mood (Martin, 2007). Unexpectedly, we found a highly comparable pattern of results for the aggressive humor-affective mood connection. This suggests that both humor styles aimed at protecting the self, regardless of whether they are displayed in a benign or potentially deleterious manner (Martin et al., 2003), can be linked to a better coping response to COVID-19. The inclination to make fun of everyday situations, including certain COVID-19-related behaviors, may contribute to re-evaluating the tragic nature of such behaviors, making it easier to laugh at them and promoting a better affective mood.

Regarding the more socially-oriented humor styles, affiliative humor was associated with higher perceived funniness of COVID-19 memes; such positive appreciation was, in turn, associated with higher happiness levels. This supports the assertion that individuals high in affiliative humor report better wellbeing as a result of their tendency to share jokes or funny comments (Martin et al., 2003), in this case internet memes. As mentioned, no relevant effects emerged for affiliative humor and perceived COVID-19 threat, which meshes well with the interpersonal nature of this humor style. Finally, SEM analyses also showed selfdefeating humor to be associated with greater COVID-19 perceived threat, which predicted 
reduced happiness and higher distress. According to Martin et al. (2003), an excessive use of self-deprecating jokes reflects, at the basic level, a negative view of the one's self and an increased tendency to suppress one's emotions. It could be assumed that higher scorers in self-defeating humor are more likely to make fun of their own behaviors during the pandemic as a way of suppressing their own negative emotions (e.g., fear, anxiety, or sadness) and that this repression may lead them to perceive COVID-19 as more life-threatening and subsequently to develop a worse affective mood.

\subsection{Limitations and Future Research Directions}

First, respondents were recruited by utilizing non-probabilistic sampling, which limits the generalizability of our findings. Second, because humor is infused with culture (Martin, 2007), the implementation of cross-cultural comparisons would provide further support to the ecological validity of these results. Third, the cross-sectional nature of our design requires us to interpret our findings as preliminary evidence, as future longitudinal investigations are needed to empirically validate the proposed models and the directionality of the observed effects. Moreover, given that the dispositional approach of this study did not include the frequency of use of humor or of sharing COVID-19 memes, further research should address this issue. Fourth, subsequent studies should seek to replicate these findings controlling for relevant aspects during a lockdown period, such as household size or types of housing. Furthermore, the small-to-moderate effect sizes observed (see Gignac \& Szodorai, 2016) lie within the range of humor-related individual differences in other studies (Martin, 2007) and can be seen as a realistic view of the relationships between humor and wellbeing (see, for example, Heintz et al., 2020). Regarding the potential practical implications of these findings, we argue that the implementation of well-established humor-based interventions aimed at minimizing the negative emotions unleashed by COVID-19 can be relevant for individuals most susceptible/vulnerable to the psychological impact of this pandemic and for professionals with a higher exposure to its tragic consequences (e.g., health professionals). Future studies should empirically validate these expectations.

\section{Conclusions}

This investigation contains empirical evidence supporting the idea that certain dispositional humorous tendencies can help individuals to cope with the adversities of the COVID-19 pandemic through how they perceive their immediate environments (i.e., perceived threat from COVID-19 and perceived funniness of COVID-19 memes). In particular, we found that humor styles aimed at protecting the self (i.e., self-enhancing and aggressive humor styles) indicated a lower perceived COVID-19 threat, which in turn was related to greater perceived funniness of COVID-19 memes, thus predicting a better affective mood.

Funding This study was supported by the FEDER/Junta de Andalucía-Consejería de Economía y Conocimiento [Proyecto B-SEJ-135-UGR18] and the Spanish Ministry of Science and Innovation [PID2019-104239GB-I00/SRA (State Research Agency/10.13039/501100011033].

Availability of Data and Material The material, data, and syntax are available at OSF (https://osf.io/u6e2n/). 


\section{Declarations}

Compliance of Ethical Standard Statement This research was conducted in accordance with the ethical guidelines of the local university and with the Ethical Standards of the 1964 Declaration of Helsinki.

Conflict of Interest/Competing Interests The authors have no conflicts of interest to declare that are relevant to the content of this article.

Informed Consent Informed consent was obtained from all participants included in the study.

\section{References}

Akram, U., Drabble, J., Cau, G., Hershaw, F., Rajenthran, A., Lowe, M. \& Ellis, J. G. (2020). Exploratory study on the role of emotion regulation in perceived valence, humour, and beneficial use of depressive internet memes in depression. Scientific Reports, 10(1), article 899. https://doi.org/10.1038/ s41598-020-57953-4

Bentler, P. M., \& Chou, C. P. (1987). Practical issues in structural modeling. Sociological Methods and Research, 16, 78-117

Bischetti, L., Canal, P., \& Bambini, V. (2020). Funny but aversive: A large-scale survey of the emotional response to Covid-19 humor in the Italian population during the lockdown. Lingua, 249, 102963. https://doi.org/10.1016/j.lingua.2020.102963

Bueno-Notivol, J., Gracia-García, P., Olaya, B., Lasheras, I., López-Antón, R., \& Santabárbara, J. (2021). Prevalence of depression during the COVID-19 outbreak: A meta-analysis of community-based studies. International Journal of Clinical and Health Psychology, 21, 100196. https://doi.org/10.1016/j. ijchp.2020.07.007

Cheng, D., Amarnani, R., Le, T., \& Restubog, S. (2019). Laughter is (powerful) medicine: The effects of humor exposure on the well-being of victims of aggression. Journal of Business and Psychology, 34, 389-402. https://doi.org/10.1007/s10869-018-9548-7

Danzer, A., Dale, J. A., \& Klions, A. H. L. (1990). Effect of exposure to humorous stimuli on induced depression. Psychological Reports, 66, 1027-1036. https://doi.org/10.2466/pr0.1990.66.3.1027

Flecha-Ortiz, J. A., Santos-Corrada, M. A., Lopez, E., \& Dones, V. (2021). Analysis of the use of memes as an exponent of collective coping during COVID-19 in Puerto Rico. Media International Australia, 178, 168-181. https://doi.org/10.1177/1329878X20966379

Freud, S. (1994). El chiste y su relación con lo inconsciente. Madrid: Alianza. (Original Work Published 1905)

Gignac, G. E., \& Szodorai, E. T. (2016). Effect size guidelines for individual differences researchers. Personality and Individual Differences, 102, 74-78. https://doi.org/10.1016/j.paid.2016.06.069

Groarke, J. M., Berry, E., Graham-Wisener, L., McKenna-Plumley, P. E., McGlinchey, E., \& Armour, C. (2020). Loneliness in the UK during the COVID-19 pandemic: Cross-sectional results from the COVID-19 Psychological Wellbeing Study. PLoS ONE, 15, e0239698. https://doi.org/10.1371/journal. pone. 0239698

González-Sanguino, C., Ausín, B., Ángel-Castellanos, M., Saiz, J., López-Gómez, A., Ugidos, C., \& Muñoz, M. (2020). Mental health consequences during the initial stage of the 2020 Coronavirus Pandemic (COVID-19) in Spain. Brain, Behavior, and Immunity, 87, 172-176. https://doi.org/10.1016/j. bbi.2020.05.040

Hampes, W. P. (2005). Correlations between humor styles and loneliness. Psychological Reports, 96, 747750. https://doi.org/10.2466/pr0.96.3.747-750

Hampes, W. P. (2010). The relation between humor styles and empathy. Europe's Journal of Psychology, 6 , 34-45. https://doi.org/10.5964/ejop.v6i3.207

Heintz, S., \& Ruch, W. (2018). Can self-defeating humor make you happy? Cognitive interviews reveal the adaptive side of the self-defeating humor style. Humor, 31, 45-472. https://doi.org/10.1515/ humor-2017-0089

Heintz, S., Ruch, W., Aykan, S., Brdar, I., Brzozowska, D., Carretero-Dios, H. \& Wong, P. S. O. (2020). Benevolent and corrective humor, life satisfaction, and broad humor dimensions: Extending the nomological network of the BenCor across 25 countries. Journal of Happiness Studies, 21, 2473-2492. https://doi.org/10.1007/s10902-019-00185-9 
Jiang, F., Lu, S., Jiang, T., \& Jia, H. (2020). Does the relation between humor styles and subjective wellbeing vary across culture and age? A meta-analysis. Frontiers in Psychology, 11, 2213. https://doi. org/10.3389/fpsyg.2020.02213

Kachanoff, F., Bigman, Y., Kapsaskis, K., \& Gray, K. (2020). Measuring realistic and symbolic threats of covid-19 and their unique impacts on well-being and adherence to public health behaviors. Social Psychological and Personality Science, 12(5), 603-616. https://doi.org/10.1177/1948550620931634

Kato, T. (2015). Frequently used coping scales: A meta-analysis. Stress and Health, 31, 315-323. https://doi. org/10.1002/smi.2557

Li, Y., Scherer, N., Felix, L., \& Kuper, H. (2021). Prevalence of depression, anxiety and post-traumatic stress disorder in health care workers during the COVID-19 pandemic: A systematic review and metaanalysis. PloS One, 16, e0246454. https://doi.org/10.1371/journal.pone.0246454

Li, S., Wang, Y., Xue, J., Zhao, N., \& Zhu, T. (2020). The impact of COVID-19 epidemic declaration on psychological consequences: a study on active Weibo users. International Journal of Environmental Research and Public Health, 17: 2032. https://doi.org/10.3390/ijerph17062032

Little, T. D., Rhemtulla, M., Gibson, K., \& Schoemann, A. M. (2013). Why the items versus parcels controversy needn't be one. Psychological Methods, 18, 285-300. https://doi.org/10.1037/a0033266

MacCallum, R. C., Browne, M. W., \& Sugawara, H. M. (1996). Power analysis and determination of sample size for covariance structure modeling. Psychological Methods, 1, 130-149. https://doi. org/10.1037/1082-989X.1.2.130

Marjanovic, Z., Greenglass, E. R., Fiksenbaum, L., \& Bell, C. M. (2013). Psychometric evaluation of the Financial Threat Scale (FTS) in the context of the great recession. Journal of Economic Psychology, 36, 1-10. https://doi.org/10.1016/j.joep.2013.02.005

Martin, R. A. (2007). The psychology of humor: An integrative approach. Elsevier

Martin, R. A., \& Lefcourt, H. M. (1983). Sense of humor as a moderator of the relation between stressors and moods. Journal of Personality and Social Psychology, 45, 1313-1324. https://doi. org/10.1037/0022-3514.45.6.1313

Martin, R. A., Puhlik-Doris, P., Larsen, G., Gray, J., \& Weir, K. (2003). Individual differences in uses of humor and their relation to psychological well-being: Development of the humor styles questionnaire. Journal of Research in Personality, 37, 48-75. https://doi.org/10.1016/S0092-6566(02)00534-2

Marrero, R. J., Carballeira, M., \& Hernández-Cabrera, J. A. (2020). Does humor mediate the relationship between positive personality and well-being? The moderating role of gender and health. Journal of Happiness Studies, 21, 1117-1144. https://oi.org/10.1007/s10902-019-00121-x

Mendiburo-Seguel, A., Páez, D., \& Martínez-Sánchez, F. (2015). Humor styles and personality: A metaanalysis of the relation between humor styles and the Big Five personality traits. Scandinavian Journal of Psychology, 56(3), 335-340. https://doi.org/10.1111/sjop.12209

Müller, L., \& Ruch, W. (2011). Humor and strengths of character. The Journal of Positive Psychology, 6, 368-376. https://doi.org/10.1080/17439760.2011.592508

Muthén, L. K., \& Muthén, B. O. (1998-2017). Mplus user’s guide (8. ed.). Los Angeles, CA: Muthén \& Muthén

Navarro-Carrillo, G., Torres-Marín, J., Corbacho-Lobato, J. M., \& Carretero-Dios, H. (2020). The effect of humour on nursing professionals' psychological well-being goes beyond the influence of empathy: A cross-sectional study. Scandinavian Journal of Caring Sciences, 34, 474-483. https://doi.org/10.1111/ scs. 12751

Navarro-Carrillo, G., Valor-Segura, I., \& Moya, M. (2021). The consequences of the perceived impact of the economic crisis on subjective well-being: The explanatory role of personal uncertainty. Current Psychology, 40(11), 5286-5300. https://doi.org/10.1007/s12144-019-00506-4

Olah, A. R., \& Ford, T. E. (2021). Humor styles predict emotional and behavioral responses to COVID-19. HUMOR, 34, 177-199. https://doi.org/10.1515/humor-2021-0009

Páez, D., Martínez-Sánchez, F., Mendiburo, A., Bobowik, M., \& Sevillano, V. (2013). Affect regulation strategies and perceived emotional adjustment for negative and positive affect: a study on anger, sadness and joy. The Journal of Positive Psychology, 8, 249-262. https://doi.org/10.1080/17439760.2013.786751

Páez, D., \& Pérez, J. A. (2020). Introduction to the special issue of social representations of Covid-19: Rethinking the pandemic's reality and social representations. Papers on Social Representations, 29, 1.1-1.24

Preacher, K. J., \& Hayes, A. F. (2008). Asymptotic and resampling strategies for assessing and comparing indirect effects in multiple mediator models. Behavior Research Methods, 40, 879-891. https://doi. org/10.3758/BRM.40.3.879 
Puente-Martínez, A., Páez, D., Ubillos-Landa, S., \& Costa-Dutra, D. (2018). Examining the structure of negative affect regulation and its association with hedonic and psychological wellbeing. Frontiers in Psychology, 9, 1592. https://doi.org/10.3389/fpsyg.2018.01592

Rossi, R., Socci, V., Talevi, D., Mensi, S., Niolu, C., Pacitti, F. \& Di Lorenzo, G. (2020). COVID-19 pandemic and lockdown measures impact on mental health among the general population in Italy. Frontiers in Psychiatry, 11, 790. https://doi.org/10.3389/fpsyt.2020.00790

Salari, N., Hosseinian-Far, A., Jalali, R., Vaisi-Raygani, A., Rasoulpoor, S., Mohammadi, M., et al. (2020). Prevalence of stress, anxiety, depression among the general population during the COVID19 pandemic: A systematic review and meta-analysis. Globalization and Health, 16, 1-11. https://oi.org/10.1186/ s12992-020-00589-w

Sanz, J. (2001). Un instrumento para evaluar la eficacia de los procedimientos de inducción de estado de ánimo: "La Escala de Valoración del Estado de Ánimo" (EVEA). Análisis y Modificación de Conducta, 27, 71-110

Schneider, M., Voracek, M., \& Tran, U. S. (2018). “A joke a day keeps the doctor away?” Meta-analytical evidence of differential associations of habitual humor styles with mental health. Scandinavian Journal of Psychology, 59, 289-300. https://doi.org/10.1111/sjop.12432

Schumacker, R. E., \& Lomax, R. G. (2010). A beginner's guide to structural equation Modeling (Third Edition). New York, NY: Taylor and Francis Group

Slater, A., Cole, N., \& Fardouly, J. (2019). The effect of exposure to parodies of thin-ideal images on young women's body image and mood. Body Image, 29, 82-89. https://doi.org/10.1016/j.bodyim.2019.03.001

Torres-Marín, J., Navarro-Carrillo, G., \& Carretero-Dios, H. (2018). Is the use of humor associated with anger management? The assessment of individual differences in humor styles in Spain. Personality and Individual Differences, 120, 193-201. https://doi.org/10.1016/j.paid.2017.08.040

Trope, Y., \& Liberman, N. (2010). Construal-level theory of psychological distance. Psychological Review, $117,440-463$

Wang, Y., Shi, L., Que, J., Lu, Q., Liu, L., Lu, Z. \& Shi, J. (2021). The impact of quarantine on mental health status among general population in China during the COVID-19 pandemic. Molecular Psychiatry, 26(9), 4813-4822. https://doi.org/10.1038/s41380-021-01019-y

Zacher, H., \& Rudolph, C. W. (2020). Individual differences and changes in subjective wellbeing during the early stages of the COVID-19 pandemic. American Psychologist. https://doi.org/10.1037/amp0000702

Publisher's Note Springer Nature remains neutral with regard to jurisdictional claims in published maps and institutional affiliations.

\section{Authors and Affiliations}

\section{Jorge Torres-Marín ${ }^{1} \cdot$ Ginés Navarro-Carrillo ${ }^{2} \cdot$ Michael Eid $^{3} \cdot$ Hugo Carretero-Dios ${ }^{1}$}

Jorge Torres-Marín

jtorresmarin@ugr.es

Michael Eid

michael.eid@fu-berlin.de

Hugo Carretero-Dios

hugocd@ugr.es

2 Department of Psychology, University of Jaén, Jaén, Spain

3 Department of Psychology, Free University of Berlin, Berlin, Germany 